\title{
Supervivencia en cáncer de mama por subtipo mediante inmunohistoquímica: Un estudio retrospectivo
}

\author{
Aura Erazo Valle-Solís ${ }^{1}$, Arturo Pabel Miranda-Aguirre', Josué Mora-Pérez², \\ Juan Antonio Pineda-Juárez $z^{3}$ Luis Ernesto Gallardo-Valencia ${ }^{4}$, Liberio Santana ${ }^{4}$, \\ Guadalupe Cervantes-Sanchez ${ }^{5}$ y Eduardo Cárdenas-Cárdenas ${ }^{*}$ \\ 'División de Padecimientos Neoplásicos y Proliferativos; ${ }^{2}$ Subdirección de Enseñanza e Investigación; ${ }^{3}$ División de Investigación Biomédica; \\ ${ }^{4}$ Dirección General; ${ }^{5}$ Servicio de Oncología Médica. Centro Médico Nacional 20 de Noviembre, Instituto de Seguridad y Servicios Sociales de los \\ Trabajadores del Estado, Ciudad de México, México
}

\section{Resumen}

Antecedentes: La clasificación del cáncer de mama en subtipos mediante la expresión de receptores hormonales (RH) y del receptor 2 del factor de crecimiento epidérmico humano (HER2) por inmunohistoquímica (IHQ) es una práctica estándar para la toma de decisiones terapéuticas. Objetivo: Conocer las características y supervivencia de cada subtipo de pacientes, que es indispensable para poder diseñar futuros estudios. Método: Realizamos un estudio retrospectivo evaluando las características clinicopatológicas y la supervivencia por subtipo mediante IHQ en mujeres con cáncer de mama. Resultados: 211 mujeres con cáncer de mama RH(+)/HER2(-), 53 con RH(+)/HER2(+), 16 con HER2(+) y 23 con RH(-)/HER2(-), con una mediana de supervivencia global en meses de 39 (20.5-62.7), 42 (25.5-65), 42 (13.7-67.7) y 26 (11-78), respectivamente, para un cociente de riesgo (HR por sus siglas en inglés, Hazard Ratio): 3.7 (IC 95\%: 1.3-10.3) en el grupo triple negativo comparado con $R H(+) / H E R 2(-)(p=0.01)$. Conclusión: Los subtipos con $R H$ positivos por $I H Q$ son los más frecuentes y este grupo de pacientes tienen una mejor supervivencia global comparada con las pacientes triple negativo.

PALABRAS CLAVE: Cáncer de mama. Inmunohistoquímica. Subtipos moleculares. Supervivencia.

\section{Abstract}

Background: Breast cancer subtype classification according to hormone receptors (HR) and human epidermal growth factor receptor 2 (HER2) using immunohistochemistry is the standard practice for therapeutic decision making. Objective: To design future studies information on characteristics and survival of each subtype is essential. Method: We conducted a retrospective study to analyze clinical and pathologic features as well as survival data according to breast cancer immunohistochemistry subtype. Results: There were 211 women with a RH(+)/HER2(-) breast cancer subtype, 53 HR(+)/HER2(+), 16 HER2(+) and 23 HR(-)/HER2(-), with a median overall survival in months of 39 (20.5-62.7), 42 (25.5-65), 42 (13.7-67.7) and 26 (11-78), respectively, for a 3.7 hazard ratio of death (95\% Confidence Interval [Cl]: 1.3-10.3) for the triple negative group as compared to the $H R(+) / H E R 2(-)$ group $(p=0.01)$. Conclusions: HR positive subtypes by immunohistochemistry where most frequent and showed a greater overall survival compared to the triple negative subtype.

KEY WORDS: Breast cancer. Immunohistochemistry. Molecular subtype. Survival.

Correspondencia:

*Eduardo Cárdenas-Cárdenas

E-mail: carcaredo84@ @otmail.com
Fecha de recepción: 06-03-2019

Fecha de aceptación: 19-03-2019

DOI: 10.24875/GMM.19005133
Gac Med Mex. 2019;155(Suppl 1):S50-S55

Disponible en PubMed www.gacetamedicademexico.com 


\section{Introducción}

El cáncer de mama es la segunda neoplasia más frecuente en el mundo y el más frecuente en las mujeres. De acuerdo con la Organización Mundial de la Salud (OMS), para el año 2018 se estimó un total de 2.1 millones de casos nuevos, lo que representó un $11.6 \%$ de todos las neoplasias. Aunado a lo anterior, la OMS también estimó para ese año un total de 626,679 muertes debidas a este padecimiento ${ }^{1}$. Asimismo, se considera la quinta causa mundial de muerte por cáncer y un problema de salud publica².

En México, el cáncer de mama se considera un problema de salud pública desde el año 2006, cuando superó al cáncer cervicouterino como la primera causa de muerte por cáncer en el país ${ }^{3}$. La incidencia actual no es bien conocida, sin embargo, cifras oficiales del Instituto Nacional de Estadística y Geografía (INEGI) reportaron que durante el 2012 se presentaron 26.6 casos por cada 100 mil mujeres mayores de 20 años de edad y durante el 2013, 26.2 casos en el mismo grupo de edad, lo que representó un $30.8 \%$ de los tumores en la mujer ${ }^{4}$. Más preocupante es el hecho que la tasa de mortalidad por cáncer de mama se ha incrementado de 8.4 muertes por 100,000 habitantes en el año 2005 a 10.1 muertes por 100,000 habitantes para el $2015^{5}$.

Actualmente, gracias a los avances en el campo de la biología molecular, el cáncer de mama se ha clasificado en cuatro subtipos moleculares que Perou, et al. ${ }^{6}$ describen basados en su expresión génica como luminal $A$, luminal $B$, sobrexpresión de receptor 2 del factor de crecimiento epidérmico humano (HER2) y basales. La evolución clínica de cada subtipo molecular del cáncer de mama es diferente y marca la pauta para el tratamiento junto con otras características clinicopatológicas, de tal forma que los luminales que se caracterizan por la expresión de receptores hormonales $(\mathrm{RH})$ y un comportamiento menos agresivo se benefician de tratamiento hormonal con o sin quimioterapia. Los que sobreexpresan HER2 tienen un comportamiento más agresivo y se benefician de terapias dirigidas como el trastuzumab y el pertuzumab en combinación con quimioterapia. Finalmente, los basaloides se tratan principalmente con quimioterapia y tienen el peor pronóstico por su comportamiento biológico y menos opciones terapéuticas comparado con los subtipos previos ${ }^{7-9}$.

El acceso a estas firmas genómicas para la clasificación del cáncer de mama es limitado, por lo que en la práctica clínica, la toma de decisiones terapéuticas se basa en una aproximación de estos subtipos mediante inmunohistoquímica (IHQ) de los RH de estrógenos, progesterona y del HER2, teniendo así los subtipos $\mathrm{RH}(+)$, HER2(-), RH(+) HER2(+), $\mathrm{RH}(-) /$ HER2(+) y $\mathrm{RH}(-) / \mathrm{HER} 2(-)$ que equivalen a luminales, luminal B HER2(+), HER2 sobreexpresado y basaloides de la clasificación molécula ${ }^{10-12}$.

Es fundamental conocer el comportamiento de estos subtipos del cáncer de mama en nuestra población para poder diseñar nuevas líneas de investigación en este grupo de pacientes, por esta razón planteamos el siguiente estudio cuyo objetivo fue realizar una revisión retrospectiva de las características clinicopatológicas por subtipo de cáncer de mama mediante IHQ y su supervivencia asociada.

\section{Método}

Se realizó un estudio de cohorte retrospectiva en el Centro Médico Nacional 20 de Noviembre, del Instituto de Seguridad y Servicios Sociales de los Trabajadores del Estado (ISSSTE), de la Ciudad de México, México. Los datos de las pacientes se obtuvieron de los registros de información y expediente clínico entre el periodo de enero de 2004 a diciembre de 2015. La aprobación del estudio y el registro de los datos fueron autorizados por los comités de investigación, ética y bioseguridad del hospital.

Se incluyeron pacientes mujeres mayores de 18 años con diagnóstico histopatológico de cáncer de mama. Las pacientes fueron identificadas conforme a la presencia o no de receptores de estrógeno, de progesterona y HER2; posteriormente se subclasificaron en los siguientes subtipos por IHQ: 1) RH(+)/HER2 (-); 2) $\mathrm{RH}(+) / \mathrm{HER} 2(+)$; 3) HER2(+), y 4) RH(-)/HER2(-). Así mismo, se analizaron variables demográficas (edad), clínicas (estadio de la enfermedad, ganglios positivos, tamaño del tumor) y tipo de tratamiento (cirugía, quimioterapia, radioterapia, hormonoterapia).

Se definió como supervivencia global a todo el tiempo trascurrido desde el diagnóstico inicial de cáncer de mama hasta la muerte del paciente y como supervivencia libre de enfermedad o progresión como todo el tiempo transcurrido desde el inicio del tratamiento hasta la recurrencia o progresión de la enfermedad.

El análisis estadístico se realizó con el programa SPSS ${ }^{\circledR}$ versión 24 (SPSS Inc., Chicago, IL, EE.UU.). Las variables continuas se presentaron como medias y desviación estándar y las variables categóricas como frecuencias absolutas y relativas. Para la 
Tabla 1. Características demográficas, clínicas y de tratamiento de la población de estudio

\begin{tabular}{|c|c|c|c|c|c|}
\hline Variable & $\begin{array}{l}\text { RH(+)/HER2(-) } \\
n=211(69.6 \%)\end{array}$ & $\begin{array}{l}\text { RH(+)/HER2(+) } \\
n=53(17.5 \%)\end{array}$ & $\begin{array}{c}\text { HER2(+) } \\
n=16(5.3 \%)\end{array}$ & $\begin{array}{c}\text { RH(-)/HER2(-) } \\
n=23(7.6 \%)\end{array}$ & $\mathbf{P}$ \\
\hline Edad, años & $53(46-62)$ & $52(45.5-58.5)$ & $51.5(44.2-56)$ & $56(43-63)$ & 0.31 \\
\hline \multicolumn{6}{|l|}{ Etapa clínica n (\%) } \\
\hline I, IIA & $77(36.4)$ & $22(41.5)$ & $3(18.7)$ & $6(26.1)$ & \\
\hline$\|\mathrm{B},\| \mathrm{A},\|\mathrm{II},\| \mathrm{IIC}$ & $97(45.9)$ & $25(47.1)$ & $11(68.5)$ & $12(52.1)$ & 0.30 \\
\hline IV & $19(9)$ & $1(1.8)$ & $1(6.2)$ & $1(4.3)$ & \\
\hline Ganglios positivos (número) & $1(0-5)$ & $1(0-4)$ & $5.5(3.7-9.2)$ & $1.5(0-4.25)$ & 0.06 \\
\hline Tamaño del tumor (mm) & $30(15-45)$ & $30(16.5-47.7)$ & $36.5(25-57.5)$ & $42.5(34-62.5)$ & 0.001 \\
\hline \multicolumn{6}{|l|}{ Tipo de tratamiento n (\%) } \\
\hline Hormonal & $174(82.5)$ & $41(77.4)$ & $4(25)$ & $7(30.4)$ & $<0.001$ \\
\hline Cirugía & $194(91.9)$ & $50(94.3)$ & $15(93.8)$ & $20(87)$ & 0.69 \\
\hline Quimioterapia & $181(85.5)$ & $47(88.7)$ & $15(93.8)$ & $23(100)$ & 0.01 \\
\hline Supervivencia global, meses & $39(20.5-62.7)$ & $42(25.5-65)$ & $42(13.7-67.7)$ & $26(11-78)$ & 0.28 \\
\hline
\end{tabular}

Las variables continuas se presentan en medianas y percentiles (p25-p75) y las variables categóricas en frecuencias y porcentajes ( $n$ y \%).

$\mathrm{RH}(+)$ : receptores hormonales positivos; $\mathrm{RH}(-)$ : receptores hormonales negativos; HER2: receptor 2 de factor epidérmico humano.

comparación de los grupos se utilizó una prueba t de Student independiente y para las variables categóricas una prueba chi cuadrada de Pearson o prueba exacta de Fisher. Para el análisis de supervivencia se utilizó la prueba de Kaplan-Meier junto con la prueba log rank. Así mismo se realizó un análisis multivariante utilizando los riesgos proporcionales de Cox para estimar la razón de riesgos con intervalos de confianza al $95 \%$ de las variables de interés (supervivencia global y supervivencia libre de progresión) ajustando los datos por variables potencialmente predictoras de mortalidad. Se consideró como estadísticamente significativa un grado de significación $(p<0.05)$.

\section{Resultados}

Se observaron un total de 303 casos de cáncer de mama. Respecto al subtipo por IHQ el $69.6 \%$ de las pacientes fueron $\mathrm{RH}(+)$ y HER2(-), el $17.5 \% \mathrm{RH}(+) /$ HER2(+), el $7.6 \%$ triple negativos, $\mathrm{RH}(-) / \mathrm{HER} 2(-)$, y el $5.3 \%$ HER2(+). Una mediana de edad de los grupos de estudio entre los 51 y 56 años $(p>0.30)$. Dentro de las variables clínicas, se apreció una mayor proporción de las etapas localmente avanzadas (IIB, IIIA, IIIB y IIIC) en comparación con las etapas tempranas (I y IIA) y metastásica (IV) en todos los grupos de estudio, sin presentar diferencias significativas $(p=0.30)$. Respecto al tipo de tratamiento recibido, se observaron diferencias estadísticamente significativas ( $p>0.001$ ) entre los grupos de estudio en la terapia hormonal con una proporción mayor en el grupo $\mathrm{RH}(+) / \mathrm{HER} 2(-)(\mathrm{n}=174)$ en comparación con los grupos $\mathrm{RH}(+) / \mathrm{HER} 2(+)(\mathrm{n}=51)$, HER2 $(+)(n=4)$ y $\mathrm{RH}(-) / \mathrm{HER} 2(-)(\mathrm{n}=7)$, así como en la terapia de cirugía $(p=0.01)$ en el grupo RH(+)/HER2(-) $(n=194)$ en comparación con los grupos $\mathrm{RH}(+) / \mathrm{HER} 2(+)$ $(n=50)$, HER2 $(+)(n=15)$ y RH(-)/HER2(-) $(n=20)$. El resto de las variables no mostraron diferencias significativas (Tabla 1).

En la Tabla 2, se muestran los riesgos no ajustados para supervivencia global en relación a la edad, etapa clínica, tipo de tratamiento y a los subtipos por IHQ; en estos últimos se observó un cociente de riesgo $(\mathrm{HR})=3$, (IC 95\%: 1.4-6.3; p < 0.01) y HR $=2.3$ (IC 95\%: 1.07-5.2; $p=0.03$ ) en los grupos $\mathrm{RH}(-) / \mathrm{HER} 2(-)$ y HER2(+), respectivamente. Posteriormente, dichos riesgos fueron ajustados (Tabla 3 ) por edad, etapa clínica y tipo de tratamiento, en donde el HR del grupo RH (-)/HER2(-) mostró un aumento (HR: 3.7; IC 95\%:1.3-10.3; $p=0.01$ ). Así mismo, se realizaron curvas de supervivencia de acuerdo con los subtipos por IHQ (log rank < 0.01) (Fig. 1).

En la tabla 2 se muestran los riesgos no ajustados para supervivencia libre de recurrencia/progresión en relación con la edad, etapa clínica, tipo de tratamiento y a los subtipos por IHQ, en estos últimos, se observó un $\mathrm{HR}=2$, (IC 95\%: 0.9-4.4; $\mathrm{p}=0.06$ ) en el grupo $\mathrm{RH}(-) /$ HER2(-). Posteriormente, dicho riesgo se ajustó por edad, etapa clínica y tipo de tratamiento (Tabla 3). 
Tabla 2. Riesgos proporcionales de Cox no ajustados para supervivencia global y supervivencia libre de recurrencia/progresión en mujeres con cáncer de mama

\begin{tabular}{|c|c|c|c|c|c|c|}
\hline \multirow[t]{2}{*}{ Variable } & \multicolumn{3}{|c|}{ Supervivencia global } & \multicolumn{3}{|c|}{ Supervivencia libre de recurrencia/progresión } \\
\hline & $\begin{array}{c}\text { HR } \\
n=303\end{array}$ & IC $95 \%$ & p & $\begin{array}{c}\text { HR } \\
n=270\end{array}$ & IC $95 \%$ & p \\
\hline \multicolumn{7}{|l|}{ Subtipos por IHQ } \\
\hline $\mathrm{RH}(+) / \mathrm{HER} 2(-)$ & 1 & --- & --- & 1 & --- & --- \\
\hline $\mathrm{RH}(+) / \mathrm{HER} 2(+)$ & 0.79 & $0.36-1.7$ & 0.56 & 1.1 & $0.5-2$ & 0.94 \\
\hline HER2(+) & 2.3 & $1.07-5.2$ & 0.03 & 1.8 & $0.8-4$ & 0.12 \\
\hline $\mathrm{RH}(-) / \mathrm{HER} 2(-)$ & 3 & $1.4-6.3$ & $<0.01$ & 2 & $0.9-4.4$ & 0.06 \\
\hline Edad (años) & 1.01 & $0.98-1.03$ & 0.32 & 0.99 & $0.96-1$ & 0.56 \\
\hline \multicolumn{7}{|l|}{ Etapa clínica n (\%) } \\
\hline I, IIA & 1 & --- & --- & 1 & --- & --- \\
\hline$\|\mathrm{B},\| \mathrm{A},\|\mathrm{I},\| \mathrm{B}, \mathrm{C}$ & 6.5 & 2.3-18.6 & $<0.01$ & 4.9 & $2.2-11$ & $<0.01$ \\
\hline IV & 30 & $10-89.7$ & $<0.01$ & 19.3 & $5.5-67.8$ & $<0.01$ \\
\hline \multicolumn{7}{|l|}{ Tipo de tratamiento } \\
\hline Hormonal & 0.40 & $0.23-0.70$ & $<0.01$ & 0.5 & $0.3-0.9$ & 0.03 \\
\hline Cirugía & 0.13 & $0.07-0.14$ & $<0.01$ & 0.13 & $0.06-0.28$ & $<0.01$ \\
\hline Quimioterapia & 1.2 & $0.49-3.1$ & 0.64 & 1.7 & $0.5-5.5$ & 0.36 \\
\hline
\end{tabular}

Las variables se presentan en razones de riesgo (HR) no ajustados e intervalos de confianza (IC) al $95 \%$.

$\mathrm{RH}(+)$ : receptores hormonales positivos; $\mathrm{RH}(-)$ : receptores hormonales negativos; HER2: receptor 2 de factor epidérmico humano; IHQ: inmunohistoquímica.

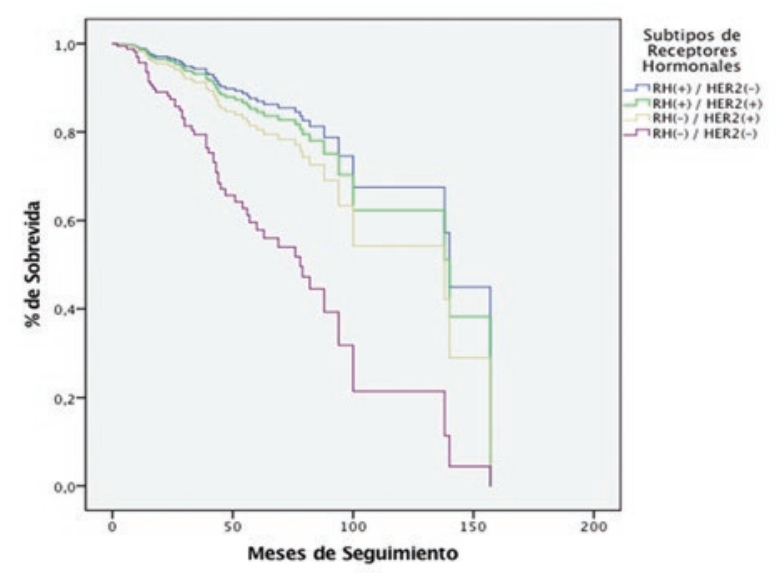

Figura 1. Curvas de Kaplan-Meier para supervivencia global de acuerdo con los subtipos de cáncer de mama por IHQ.

$R H(+) / H E R 2(-)$

$R H(+) / H E R 2(+)$

$R H(-) / H E R 2(+)$

$R H(-) / H E R 2(-)$

Meses de seguimiento

\section{Discusión}

Nuestros resultados muestran diferencias en la supervivencia global en las pacientes con cáncer de mama por subtipo inmunohistoquímico. Observamos que son más frecuentes las pacientes con cáncer de mama $\mathrm{RH}(+)$, las cuales se presentan significativamente con un menor tamaño tumoral y con un menor número de ganglios, lo cual es reflejo de un comportamiento biológico menos agresivo y por ende con mayor supervivencia global. En contraste, las pacientes que presentan cáncer de mama con un subtipo triple negativo, que aun cuando menos frecuente, se presentan al diagnóstico con tumores de mayor tamaño y un peor pronóstico, con una menor supervivencia global (HR: 3.7 ajustado; IC 95\%: 1.3-10.3).

En estudios con población europea, asiática y de los Estados Unidos se observan resultados similares a los nuestros, con una mayor proporción de pacientes con $\mathrm{RH}(+)$ y una mejor supervivencia global en este subgrupo de pacientes; sin embargo, nuestra proporción de pacientes HER2(+), con o sin $\mathrm{RH}$, fue mayor, con un $25 \%$ comparado con un $17 \%$ o menos reportada por ellos ${ }^{13-18}$. Históricamente los pacientes con HER2(+) tenían un mal pronóstico ${ }^{9}$, pero con los avances en terapias dirigidas para este subtipo de cáncer de mama, se ha alcanzado supervivencias similares al grupo de $\mathrm{RH}(+) / \mathrm{HER} 2(-)^{19}$. Así mismo, contrasta el porcentaje de pacientes con enfermedad localmente avanzada, que en nuestro estudio es 
Tabla 3. Riesgos proporcionales de Cox ajustados para supervivencia global y supervivencia libre de recurrencia/ progresión en mujeres con cáncer de mama

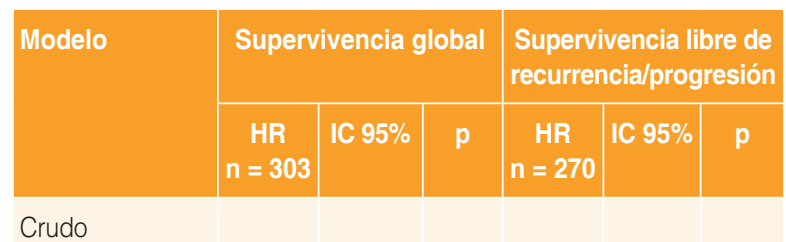

Subtipos por $\mathrm{IHQ}$

\begin{tabular}{|l|c|c|c|c|c|c|}
\hline RH(+)/HER2(-) & 1 & --- & -- & 1 & --- & --- \\
\hline$R H(+) / H E R 2(+)$ & 0.79 & $0.36-1.7$ & 0.56 & 1.1 & $0.5-2$ & 0.94 \\
\hline HER2(+) & 2.3 & $1.07-5.2$ & 0.03 & 1.8 & $0.8-4$ & 0.12 \\
\hline RH(-)/HER2(-) & 3 & $1.4-6.3$ & $<0.01$ & 2 & $0.9-4.4$ & 0.06 \\
\hline
\end{tabular}

Ajustado

Subtipos por $\mathrm{IHQ}$

\begin{tabular}{l|c|c|c|c|c|c|}
\hline $\mathrm{RH}(+) / \mathrm{HER} 2(-)$ & 1 & --- & -- & 1 & --- & --- \\
\hline $\mathrm{RH}(+) / \mathrm{HER} 2(+)$ & 1.1 & $0.49-2.8$ & 0.70 & 1.2 & $0.5-2.6$ & 0.64 \\
\hline HER2(+) & 1.5 & $0.53-4.3$ & 0.42 & 1.3 & $0.4-3.4$ & 0.59 \\
\hline $\mathrm{RH}(-) / \mathrm{HER} 2(-)$ & 3.7 & $1.3-10.3$ & 0.01 & 1.6 & $0.6-4.7$ & 0.32
\end{tabular}

Los variables se presentan razones de riesgo (HR) ajustados por edad, etapa clínica, tratamiento por quimioterapia, tratamiento por cirugía y tratamiento por hormonoterapia e intervalos de confianza (IC) al 95\%.

$\mathrm{RH}(+)$ : receptores hormonales positivos; $\mathrm{RH}(-)$ : receptores hormonales negativos; HER2: receptor 2 de factor epidérmico humano; IHQ: inmunohistoquímica.

mayor al $50 \%$ de los casos comparado con menos del $20 \%$ observado en países desarrollados ${ }^{13-15,17}$.

Respecto a otros estudios en población mexicana, el estudio más grande reportado es en una cohorte de pacientes del Seguro Popular ${ }^{20}$, en el cual de 4,300 mujeres el $60.7 \%$ eran $\mathrm{RH}(+) / \mathrm{HER} 2(-)$ y tenían un mejor pronóstico comparado con mujeres con cáncer de mama triple negativo con un $\mathrm{HR}=2.16$ (IC 95\%: 1.69-2.75). La frecuencia de cáncer de mama HER2(+) es similar a la nuestra, con un $23 \%$ y de igual manera son más frecuentes los estadios avanzados de la enfermedad.

Un estudio en un hospital del Instituto Mexicano del Seguro Social (IMSS) por Pérez-Rodríguez ${ }^{21}$ reportó una proporción de cáncer de mama luminal $\mathrm{A}$ del $65 \%$ y luminal $B$ del $12 \%$, lo cual concuerda con nuestros resultados, siendo los subtipos luminales con expresión de $\mathrm{RH}$ los más frecuentes; sin embargo la proporción de triple negativos del $14 \%$ fue mayor a la reportada por nosotros, del $7.6 \%$. Las diferencias en supervivencia global reportadas en este estudio fueron similares a las observadas por nosotros, siendo mayor en las que expresaban $\mathrm{RH}$ comparados con las que no expresaban $\mathrm{RH}$.
Este es el primer estudio que describe las características y supervivencia asociada de los diferentes subtipos mediante IHQ del cáncer de mama en mujeres derechohabientes del ISSSTE, lo cual suma información importante junto con los estudios en población del Seguro Popular e IMSS para tener una visión más completa de la realidad de esta enfermedad en México. El cáncer de mama es una enfermedad curable si se diagnostica en etapas tempranas, por lo que es fundamental mejorar los programas de tamizaje, diagnóstico y referencia de estas pacientes, para poder disminuir la frecuencia de casos en etapas avanzadas de la enfermedad, las cuales siguen siendo las más frecuentes en nuestra población.

La principal debilidad de nuestro estudio es que la muestra es pequeña y derivada de un hospital de concentración de tercer nivel, por lo que el sesgo de selección de los pacientes no permite extrapolar los resultados a la situación de todo el instituto. Los subtipos de cáncer de mama por IHQ permiten aproximar la clasificación molecular mediante firmas genómicas descritas por Perou, et al. ${ }^{6}$. El uso de IHQ se considera un estándar en el abordaje del cáncer de mama, al brindar una aproximación a los subtipos moleculares del cáncer de mama, sin embargo el acceso a las firmas genómicas necesarias para la clasificación molecular es limitado, por lo que en la práctica el uso de $\mathrm{IHQ}$ es fundamental ${ }^{12,13}$.

En conclusión, la mayor proporción de mujeres con cáncer de mama en nuestra población derechohabiente presentan un subtipo por IHQ con expresión de $\mathrm{RH}$ y este grupo de pacientes tienen una mejor supervivencia global comparada con las pacientes triple negativo. El desarrollo de nuevas opciones terapéuticas para este grupo de mal pronóstico es fundamental, buscando lograr un impacto como el que se ha logrado en las pacientes HER2 positivo.

\section{Bibliografía}

1. Bray F, Ferlay J, Soerjomataram I, Siegel RL, Torre LA, Jemal A. Global cancer statistics 2018: GLOBOCAN estimates of incidence and mortality worldwide for 36 cancers in 185 countries. CA Cancer J Clin. 2018;68:394-424.

2. Tao Z, Shi A, Lu C, Song T, Zhang Z, Zhao J. Breast cancer: Epidemiology and etiology. Cell Biochem Biophys. 2015:72:333-8.

3. Rodríguez Cuevas S, Capurso García M. Epidemiología del cáncer de mama. Ginecol Obstet Mex. 2006;74:585-93.

4. Estadísticas vitales. Defunciones generales y fetales [Internet]. México: INEGI 2016. Disponible en: http://www3.inegi.org.mx/rnm/index.php/catalog/303

5. Aldaco-Sarvide F, Pérez-Pérez P, Cervantes-Sánchez G, Torrecillas-Torres L, Argentina Erazo-Valle-Solís A, Cabrera P, et al. Mortalidad por cáncer en México: actualización 2015. Gac Mex Oncol. 2008;17:28-34.

6. Perou CM, Sorlie T, Eisen MB, van de Rijn M, Jeffrey SS, Rees CA, et al. Molecular portraits of human breast tumours. Nature. 2000;406:747-52.

7. Wishart GC, Bajdik CD, Azzato EM, Dicks E, Greenberg DC, Rashbass J, et al. A population-based validation of the prognostic model PREDICT for early breast cancer. Eur J Surg Oncol. 2011;37:411-7. 
8. Schmidt S, Monk JM, Robinson LE, Mourtzakis M. The integrative role of leptin, oestrogen and the insulin family in obesity-associated breast cancer: potential effects of exercise. Obes Rev. 2015;16:473-87.

9. Carey LA, Perou CM, Livasy CA, Dressler LG, Cowan D, Conway K et al. Race, breast cancer subtypes, and survival in the Carolina Breast Cancer Study. JAMA. 2006;295:2492-502.

10. Bhargava R, Striebel J, Beriwal S, Flickinger JC, Onisko A, Ahrendt G et al. Prevalence, morphologic features and proliferation indices of breast carcinoma molecular classes using immunohistochemical surrogate markers. Int J Clin Exp Pathol. 2009;2:444-55.

11. Rakha EA, El-Sayed ME, Reis-Filho JS, Ellis IO. Expression profiling technology: its contribution to our understanding of breast cancer. Histopathology. 2008;52:67-81

12. Goldhirsch A, Winer EP, Coates AS, Gelber RD, Piccart-Gebhart M, Thurlimann B, et al. Personalizing the treatment of women with early breas cancer: highlights of the St Gallen International Expert Consensus on the Primary Therapy of Early Breast Cancer 2013. Ann Oncol. 2013;24:2206-23.

13. Park S, Koo JS, Kim MS, Park HS, Lee JS, Lee JS, et al. Characteristics and outcomes according to molecular subtypes of breast cancer as classified by a panel of four biomarkers using immunohistochemistry. Breast. 2012;21:50-7.

14. Engstrom MJ, Opdahl S, Hagen Al, Romundstad PR, Akslen LA Haugen OA, et al. Molecular subtypes, histopathological grade and survival in a historic cohort of breast cancer patients. Breast Cancer Res Treat. 2013;140:463-73.
15. Onitilo AA, Engel JM, Greenlee RT, Mukesh BN. Breast cancer subtypes based on ER/PR and Her2 expression: comparison of clinicopathologic features and survival. Clin Med Res. 2009;7:4-13.

16. Spitale A, Mazzola P, Soldini D, Mazzucchelli L, Bordoni A. Breas cancer classification according to immunohistochemical markers: clinicopathologic features and short-term survival analysis in a population-based study from the South of Switzerland. Ann Oncol. 2009; 20;628-35.

17. Parise CA, Caggiano V. Breast cancer survival defined by the ER/PR/ HER2 subtypes and a surrogate classification according to tumor grade and immunohistochemical biomarkers. J Cancer Epidemiol. 2014;2014:11.

18. Blows FM, Driver KE, Schmidt MK, Broeks A, van Leeuwen FE, Wesseling $\mathrm{J}$, et al. Subtyping of breast cancer by immunohistochemistry to investigate a relationship between subtype and short and long term survival: a collaborative analysis of data for 10,159 cases from 12 studies. PLoS Med. 2010;7:e1000279.

19. Incorvati JA, Shah S, Mu Y, Lu J. Targeted therapy for HER2 positive breast cancer. J Hematol Oncol. 2013:6:38.

20. Reynoso-Noverón N, Villarreal-Garza C, Soto-Perez-de-Celis E, Arce-Salinas C, Matus-Santos J, Ramírez-Ugalde MT, et al. Clinical and epidemiological profile of breast cancer in Mexico: Results of the Seguro Popular. J Glob Oncol. 2017:3:757-64.

21. Pérez-Rodríguez G. [Prevalence of breast cancer sub-types by immunohistochemistry in patients in the Regional General Hospital 72, Instituto Mexicano del Seguro Social]. Cir Ciruj. 2015;83:193-8. 